RESEARCH AND PRACTICE

\title{
Racial and socio-economic disparities in melanoma incidence rates in Georgia: 2000-2011
}

\author{
MaryBeth Culp ${ }^{1}$, MPH, Sara Wagner Robb ${ }^{1}$, PhD, A. Rana Bayakly², MPH, John E. Vena ${ }^{3}$, PhD
}

${ }^{1}$ Department of Epidemiology and Biostatistics, College of Public Health, University of Georgia, Athens, GA; ${ }^{2}$ Georgia Comprehensive Cancer Registry, Georgia Department of Public Health, Atlanta, GA; ${ }^{3}$ Department of Public Health Sciences, School of Medicine, Medical University of South Carolina, Charleston, SC

\begin{abstract}
Background: The objective of this research was to investigate melanoma incidence rates and health outcomes in Georgia over time and by race, socio-economic status (SES), and gender.

Methods: Age-adjusted melanoma incidence rates were obtained from the Georgia Comprehensive Cancer Registry SEER*Stat Database (2000-2011). To compare incidence rates across counties, and public health districts and by race, SES and gender, maps were generated using Geographic Information Systems (GIS). A cluster analysis was performed by use of SaTScan, and maps were created to visualize clusters of melanoma cases.

Results: In Georgia, from 2000-2011, age-adjusted incidence rates for melanoma were higher among Whites than Blacks (28.0 vs. 1.1 per 100,000 population). For both races, high rates were found to be associated with high SES. For Whites, high rates were concentrated in urban areas relative to Blacks in rural areas. Clusters of melanoma incident cases were found mainly in the north central region of Georgia.

Conclusions: For Georgia, results for map comparisons are consistent with previous research findings that higher melanoma incidence rates are associated with high SES for Whites and, to a lesser extent, for Blacks. Melanoma interventions in Georgia should focus on urban White and rural Black at-risk populations, especially those with high SES.
\end{abstract}

Keywords: melanoma; racial disparities; socioeconomic status (SES); Geographic Information Systems (GIS); cluster analysis

\section{INTRODUCTION}

Skin cancer is the most frequently diagnosed cancer in the United States (US) with melanoma, the malignant form accounting for $75 \%$ of all skin cancer deaths (Shoo and Kashani-Sabet, 2009). Melanoma is a highly preventable form of cancer that, if caught at an early stage, can be treated with promising results. From 1992 to 2006, melanoma incidence rates among non-Hispanic Whites increased for all ages, but mortality rates increased only among persons >65 years old (Jemal et al, 2011). Educational campaigns across the US to promote awareness about melanoma have helped to slow the rising incidence of melanoma, although disparities still exist among racial groups and by socio-economic status (SES) (Giblin and Thomas, 2007).

In Georgia (GA), from 2002-2006, the rate of new melanoma diagnoses was 13\% higher than the national average (Office of Air and Radiation, 2010). Furthermore, in this period, White County, located in the northeastern region of GA, had the second highest incidence of melanoma among counties nationwide (Singh et al, 2011). Despite these observations, only limited epidemiological research on melanoma in GA has been conducted, making descriptions of geographic and racial trends a priority.

The incidence of melanoma in non-Hispanic Whites is higher than among ethnic minority populations; the lifetime risk of developing melanoma is 23 times higher among Whites than among Blacks (Boscoe et al, 2014; Shoo and Kashani-Sabet, 2009; Singh et al, 2011; American Cancer Society, 2013). Among ethnic minorities, the rarity of melanoma occurrence and atypical presentations lead to delayed diagnoses at later stages and poorer clinical outcomes (Harvey et al, 2014; Hu et al, 2014; Shoo and Kashani-Sabet, 2009). In GA from 2002-2006, melanoma incidence rates for Whites were the $6^{\text {th }}$ highest in the US (Office of Air and Radiation, 2010). The population of GA is $62.8 \%$ White (US 77.9\%) and $31.2 \%$ Black (relative to $13.1 \%$ in the US), which provides a large sample size for statistical power in evaluating racial comparisons (US Census Bureau, 2010).

International studies have shown an association between high SES and an increased risk for melanoma (Aase and Bentham, 1996; Aarts et al, 2010; Van der Aa et al, 2011). In the US, high SES is associated with a higher incidence 
(Boscoe et al, 2014; Clegg et al, 2009). However, individuals with lower educational achievement and low SES have a decreased melanoma risk perception, less knowledge about detection, and lower rates of patientphysician communication (Pollitt et al, 2012). These discrepancies among individuals with low SES contribute to late-stage diagnoses of melanoma and to poorer clinical outcomes relative to those with high SES, who are typically diagnosed earlier in disease progression (Mandala et al, 2011; Youl et al, 2011; Zell et al, 2008). Further, lower SES has been associated with a higher age- and sex-adjusted mortality/incidence ratio for melanoma (0.37 vs. 0.25$)$, which is indicative of worse survival (Geller et al, 1996). Although melanoma incidence rates are higher among individuals with a high SES, outcomes are typically worse among those with a low SES. In GA, the median household income from 2008-2012 was lower than that for the US (\$49,604 vs. \$53,046) (U.S. Census Bureau, 2010). Therefore, the effects of SES on health outcomes, particularly as they relate to delayed diagnoses of melanoma, may be pronounced in GA, especially relative to the rest of the US.

The objective of this research was to investigate melanoma incidence rates and health outcomes in GA by race, SES, and gender. This was accomplished by exploring the descriptive statistics of the disease over time as well as geographically. Disparities in incidence rates and stage at diagnosis between races were compared at the public health district level and statewide. Geographic clusters of melanoma incident cases were also identified at the county and census tract level, after adjusting for race and age. Limited research on melanoma in GA has been conducted, making initial epidemiologic descriptions of geographic trends relevant for the development of research studies and for exploring population- and/or geography-specific interventions.

\section{METHODS}

Melanoma incidence data for the years 2000-2011 were obtained from the Georgia Comprehensive Cancer Registry (GCCR) SEER*Stat Database (Surveillance Research Program, National Cancer Institute SEER*Stat software (www.seer.cancer.gov/seerstat version 8.2.1). The GCCR, a statewide, population-based cancer registry that collects information on all cancer cases diagnosed among GA residents, is a participant in the National Program for Cancer Registries and the North American Association of Central Cancer Registries. The GCCR meets national standards for cancer registration and is gold-certified with high ratings for data quality and representativeness. All rates were age-adjusted to the 2000 United States Standard Population and expressed per 100,000 population. Stage of melanoma at diagnosis was compared between Whites and Blacks using the SEER*Stat Summary Stage 2000 (Young et al, eds, 2001). These data were divided into four disease stages: localized (cells limited to organ of origin), regional (cells traveled beyond organ of origin), distant (cells growing in a new area of the body), and unknown/unstaged (information not given). Localized tumors were defined as early-stage disease; regional and distant tumors were classified as late-stage disease. Because limited data were available on other races in SEER*Stat the study was limited to Whites and Blacks.

Age-adjusted melanoma incidence rates (IR) per 100,000 were obtained and evaluated by race (Whites vs. Blacks), gender, stage (early vs. late), county ( $\mathrm{N}=159)$, public health district ( $\mathrm{N}=18)$, and over time (2000-2011). Hot spots of melanoma incidence were also analyzed at the county level for all races and among Whites only using the Getis-Ord $\mathrm{Gi}^{*}$ Statistic in Geographic Information Systems (GIS ArcMap software, version 10.1; ESRI, Redlands, GA). The Getis-Ord Gi* Statistic is an analysis tool that uses spatial data to detect statistically significant clusters of high values (hot spots) and low values (cold spots) (Environmental Systems Research Institute, 2014). There were insufficient data at the county level to perform this analysis for Blacks. $\mathrm{Z}$-scores, indicative of the amount of spatial clustering, and $\mathrm{p}$-values were generated for each GA county.

For 2000-2011 data, SaTScan ${ }^{\mathrm{TM}}$ software, version 9.3.1 (Martin Kulldorff, Harvard Medical School, Boston, and Information Management Services Inc, Calverton, Maryland) was utilized to examine clustering of melanoma incidence. Clusters were determined by "gradually scanning a window across time and/or space, noting the number of observed and expected observations inside the window at each location” (Kulldorff, 2015). Melanoma incidence clusters were tested using the discrete Poisson model, which considered counts of melanomas per GA census tract $(\mathrm{N}=1,624)$. For all models, a purely spatial circular scan statistic, 50\% spatial scanning window, and 999 simulations were used. All cluster analyses were adjusted for census tract population, race, and age with a 19category age variable consistent with the GCCR's classification schema. Clusters were considered as significant at $\mathrm{p}<0.1$.

Public health districts in GA were dichotomized as low and high SES based on the median per capita income among the public health districts compared to the overall median value of the median per capita incomes of the public health districts (\$20,005) (U.S. Census Bureau, 2010). GA counties were ranked by SES based on their median per capita income compared to the median per capita income of GA $(\$ 18,502)$. Counties with median per capita incomes greater than or equal to the median per capita income of GA were characterized as high SES. Incidence data were examined by use of Microsoft Excel (2007; Microsoft Corporation, Redmond, WA). Maps were generated using GIS to compare incidence rates across counties and public health districts, and by race, SES and gender. GIS was also used to visualize the results of the hotspot and cluster analyses.

\section{RESULTS}

From 2000 to 2011 among individuals of all races, the incidence rate of melanoma was slightly higher in GA relative to the rest of the US (21.0 vs. 20.3 per 100,000 population; Table 1). In GA, age-adjusted incidence rates of melanoma were significantly higher among Whites than 
Blacks (28.0 vs. 1.1; Table 1), which is consistent with national trends. During this same ten-year time period, melanoma incidence rates among Whites have been steadily increasing (from 22.3 to 28.0), whereas rates for Blacks have remained constant (average of 1.1; Figure 1). Furthermore, among Whites in GA, melanoma incidence rates were higher for males than females (35.7 vs. 22.7; Table 1). From 2000 to 2011, incidence rates for both genders increased although males had higher rates than females and experienced a higher increase (data not shown). For both races from 2000 to 2011, the predominant stage at melanoma diagnosis was localized. Approximately $85 \%$ of melanoma diagnoses in Whites were early-stage; only $10 \%$ were late-stage. In contrast, among Blacks, 58\% were early-stage, and 34\% were late-stage.

\section{Table 1. Overall Melanoma Incidence Rates for GA and the US by Race and Gender, 2000-2011}

\begin{tabular}{|c|c|c|c|}
\hline & & \multicolumn{2}{|c|}{ Incidence Rate (CI $\mathbf{I}^{\mathbf{a}}$} \\
\hline Race & Gender & GA & United States \\
\hline All & & $21.0(20.7-21.3)$ & $26.3(20.2-20.4)$ \\
\hline & Male & $27.9(27.4-28.4)$ & $16.1(16.0-16.2)$ \\
\hline & Female & $16.3(16.0-16.7)$ & $24.0(23.9-24.1)$ \\
\hline White & & $28.0(27.6-28.4)$ & $30.6(30.4-30.8)$ \\
\hline & Male & $35.7(35.1-36.4)$ & $19.3(19.2-19.4)$ \\
\hline & Female & $22.7(22.2-23.2)$ & $1.0(0.9-1.1)$ \\
\hline Black & & $1.1(0.9-1.2)$ & $1.1(1.0-1.2)$ \\
\hline & Male & $1.3(1.0-1.6)$ & $1.0(0.9-1.0)$ \\
\hline
\end{tabular}

Rates are per 100,000 and age-adjusted to the 2000 US Standard Population (19 age groups - Census P25-1130) standard. *95\% Confidence Interval

Figure 1. Melanoma Incidence Rates in GA from 2000-2011 by Race and Gender

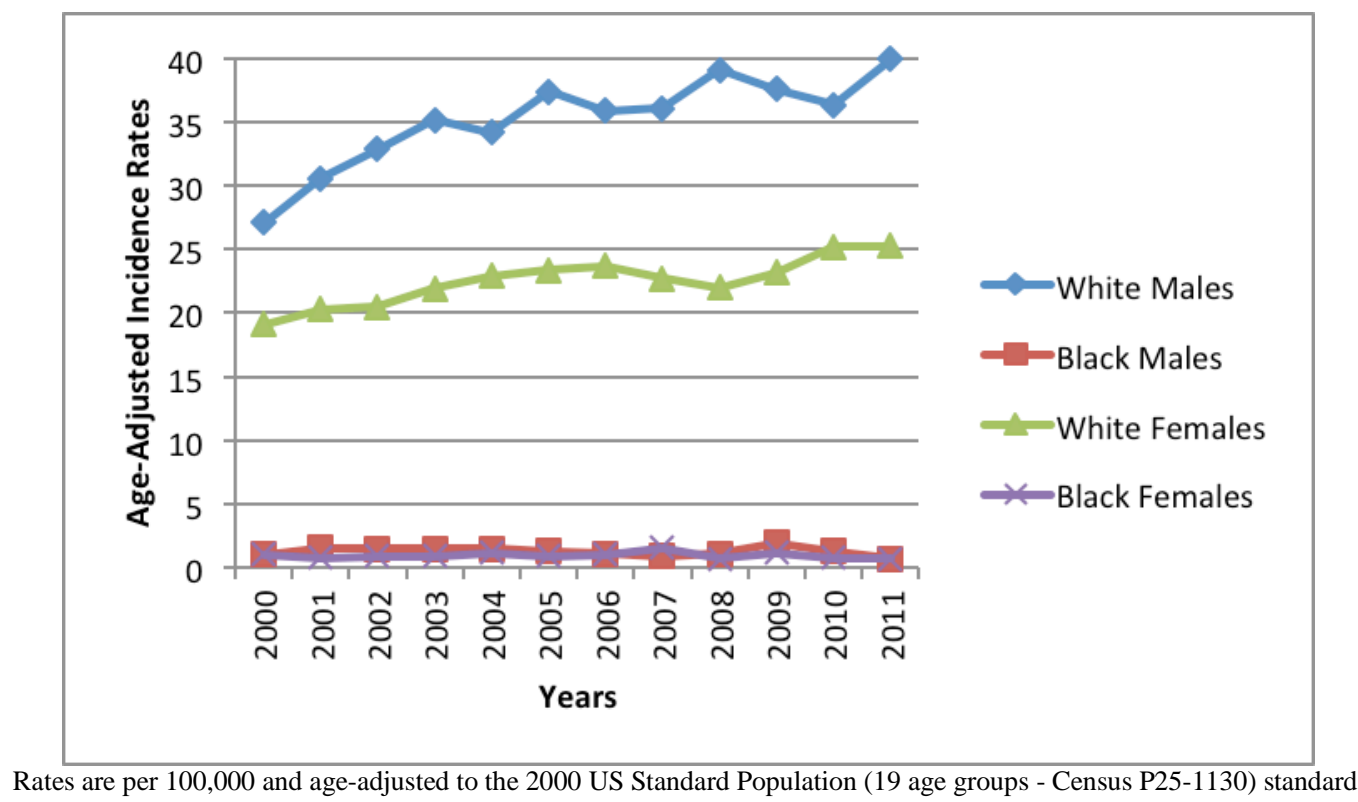

GA has 18 public health districts that are each comprised of one or more of GA's 159 counties and county health departments. These districts are numbered from 1-1 to 10 based loosely on geography from north to south and east to west. All but one (9-1) of the public health districts with a high SES $(>\$ 20,005)$ was located in the northern part of GA
(Figure 2). Only one district (1-1) in the northern region of GA was classified as low SES $(<\$ 20,005)$ (Figure 2$)$. The highest incidence rates for Whites were in districts 3-2 (44.3), 3-1 (36.5), and 3-5 (35.4), which are the three districts encompassing the city of Atlanta (Fulton, CobbDouglas, and DeKalb, respectively) (Figure 2). The highest 
incidence rates for Blacks were in districts 2 (2.7), 1-1 (2.4), and 8-1 (2.3), which are located in Northeast (Gainesville), Northwest (Rome), and South (Valdosta) areas of GA (Figure 2). Of the 18 public health districts in GA, eight had high melanoma incidence rates $(>23.7$, the median of the incidence rates by public health district among Whites), and a high SES for Whites. Among Blacks, only four districts had high melanoma incidence rates ( $>1.2)$ and a high SES. Of the 159 counties in GA, 57 had high melanoma incidence rates among Whites and high SES for both genders (data not shown). For both males and females, 55 counties had high melanoma incidence rates and high SES.

Figure 2. Melanoma Incidence Rates by Public Health District for Whites and Blacks 2000-2011
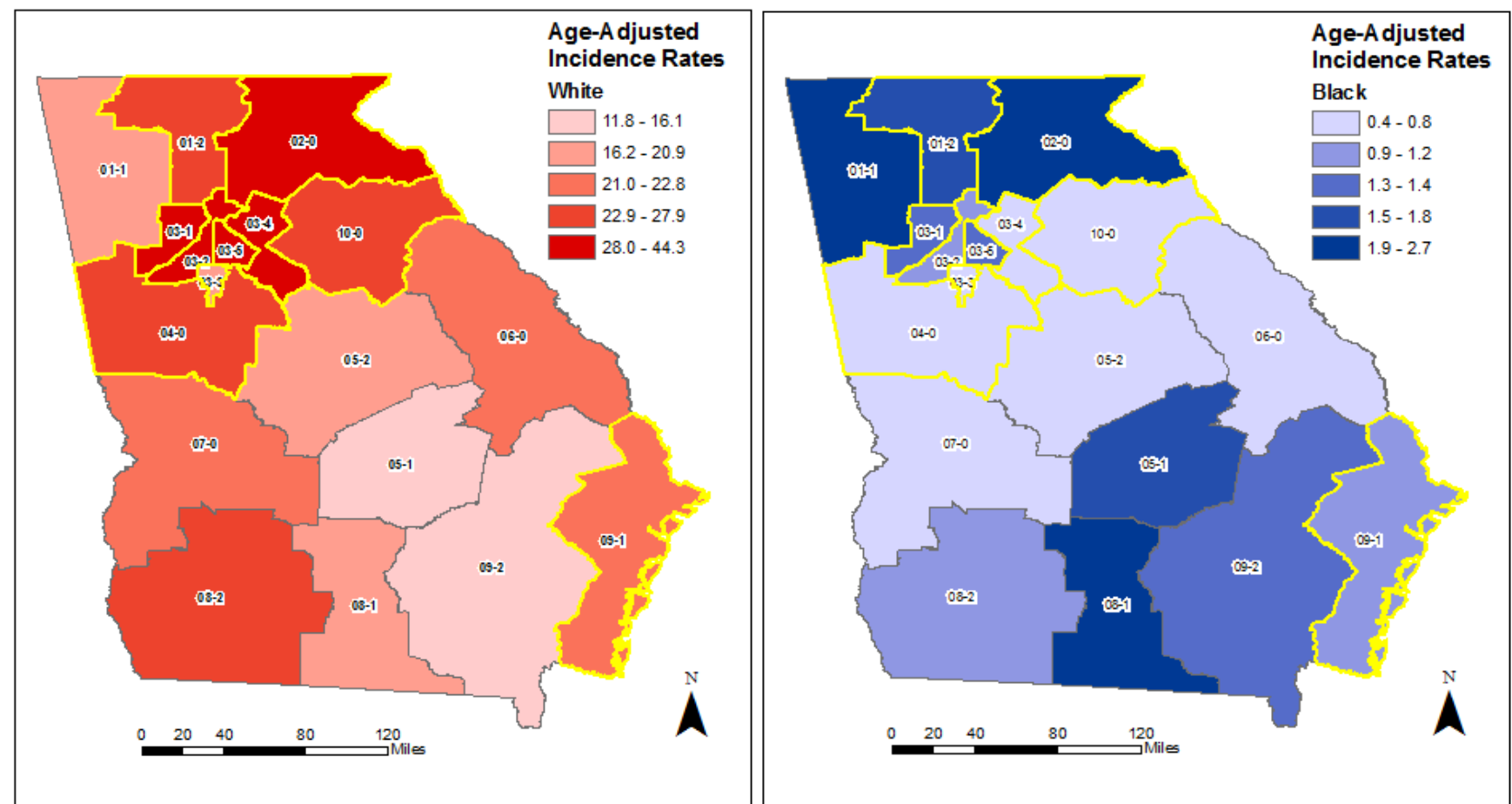

Data Source: Georgia Comprehensive Cancer Registry SEER*Stat Database.

*Rates are per 100,000 and age-adjusted to the 2000 US Standard Population (19 age groups - Census P25-1130) standard.

**Highlighted areas are categorized as high SES compared to the median of the median per capita income of the Public Health districts $(\$ 20,005)$.

*** The public health district names are as follows: 1-1 Northwest; 1-2 Northwest Georgia; 2 North; 3-1 Cobb-Douglas; 3-2 Fulton; 3-3 Clayton; 3-4 East Metro; 3-5 DeKalb; 4 LaGrange; 5-1 South Central; 5-2 North Central; 6 East Central; 7 West Central; 8-1 South; 8-2 Southwest; 9-1 Coastal; 9-2 Southeast; 10 Northeast.

Figure 3 depicts county-level hotspots for all races and for Whites from 2000 to 2011 as determined by the Getis-Ord $\mathrm{Gi}^{*}$ statistic. There were insufficient data to perform this analysis at the county level for Blacks. For both races, the geographical trends mentioned previously were confirmed with statistically significant $(\mathrm{p}<0.05)$ hot spots (areas with high melanoma incidence rates) in 27 (93\% of counties in the hotspot; $17 \%$ of all GA counties) of the northern counties of GA (Figure 3). There were 18 (69\%; 11\%) statistically significant values for counties identified as cold spots (areas with low melanoma incidence rates) in the central area of GA (Figure 3). Among Whites, the hot and cold spots were similar to those for all races. There were 23 counties in the statistically significant hot spot in the northern region of GA and one statistically significant county in a hot spot in the southern area (Figure 3). Alternatively, there were 13 counties in statistically significant cold spots located in the central and eastern portions of GA (Figure 3). 
Figure 3. Getis-Ord Gi* Statistic for hot spot analysis of melanoma incidence for all races and among whites by county, 2000-2011

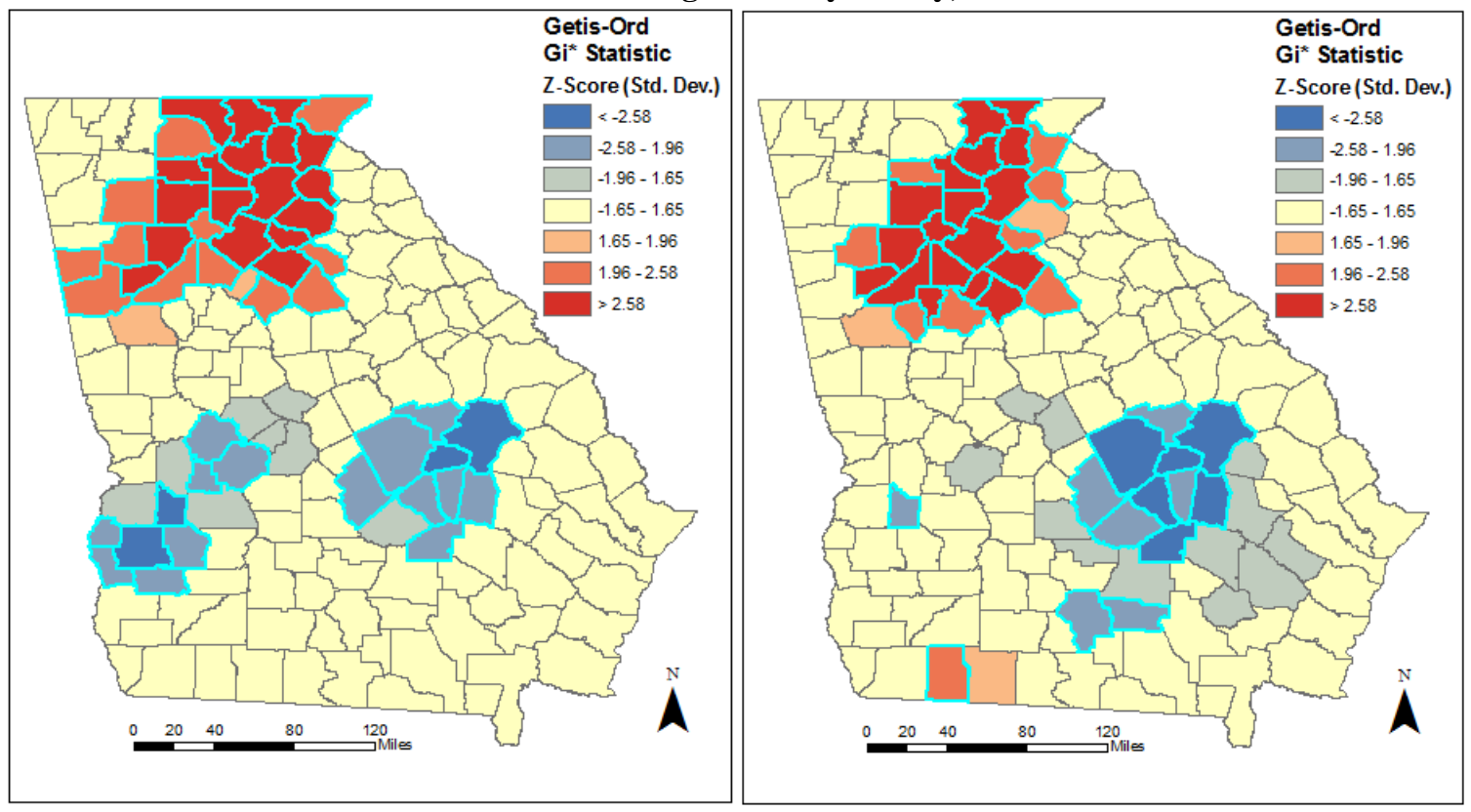

Positive Z-score indicates clustering of high values. Negative Z-score indicates clustering of low values. Highlighted area indicates statistically significant cluster, $\mathrm{p}<0.05$.

Cluster analysis of all melanoma cases by census tract in GA from 2000-2011 among Whites and Blacks revealed 20 statistically significant $(\mathrm{p}<0.05)$ clusters of melanoma incident cases and four other non-statistically significant clusters (Figure 4; Table 2). The largest cluster was centered in DeKalb county, which is located in the North central area of GA (cluster number 1; $74.61 \mathrm{~km}$ radius; $\mathrm{p}<0.000001$; Figure 4; Table 2). The second largest cluster was centered in a Northeastern county of Georgia, Union County (cluster number 10; $31.48 \mathrm{~km}$ radius; $\mathrm{p}<0.000001$; Figure 4; Table 2). (Since cluster models estimate a census tract as the geographic centroid or reference location, a cluster radius of zero indicates a cluster localized to one specific census tract.) There were three such statistically significant $(\mathrm{p}<0.05)$ clusters with radii of zero: Chatham County (cluster 14; $\mathrm{p}=0.000019)$, Greene County (cluster 15; $\mathrm{p}=$ 0.000358) and Columbia county (cluster 20; $\mathrm{p}=0.041$ ).

Figure 4. Clusters of age-adjusted melanoma incident cases for both races, 2000-2011

SaTScan Parameters: Discrete Poisson model adjusted for age $(\mathrm{N}=43,559) .50 \%$ spatial scanning window, 999 simulations. Data Source: Georgia Comprehensive Cancer Registry 
Table 2. Clusters of census tract-level melanoma incidence for both races, 2000-2011

\begin{tabular}{|c|c|c|c|c|c|}
\hline Cluster Number $^{\mathrm{a}}$ & Census Tract $^{b}$ & County $^{c}$ & Radius $^{d}$ & p value & Relative risk $^{\mathrm{ef}}$ \\
\hline 1 & 021208 & DeKalb & 74.61 & $<0.000001$ & 2.0 \\
\hline 2 & 130600 & Forsyth & 16.18 & $<0.000001$ & 2.3 \\
\hline 3 & 091001 & Cherokee & 14.62 & $<0.000001$ & 1.7 \\
\hline 4 & 010109 & Fulton & 8.57 & $<0.000001$ & 1.7 \\
\hline 5 & 120201 & Paulding & 16.28 & $<0.000001$ & 1.7 \\
\hline 6 & 001200 & Hall & 24.68 & $<0.000001$ & 1.6 \\
\hline 7 & 110501 & Walton & 14.39 & $<0.000001$ & 1.7 \\
\hline 8 & 008702 & Fulton & 8.83 & $<0.000001$ & 1.6 \\
\hline 9 & 140303 & Fayette & 15.79 & $<0.000001$ & 1.5 \\
\hline 10 & 990202 & Union & 31.48 & $<0.000001$ & 1.4 \\
\hline 11 & 070203 & Henry & 15.77 & $<0.000001$ & 1.4 \\
\hline 12 & 050715 & Gwinnett & 5.04 & $<0.000001$ & 1.4 \\
\hline 13 & 980300 & Harris & 16.33 & 0.000005 & 1.7 \\
\hline 14 & 011002 & Chatham & 0.00 & 0.000019 & 1.7 \\
\hline 15 & 950300 & Greene & 0.00 & 0.000038 & 2.1 \\
\hline 16 & 080402 & Douglas & 14.10 & 0.000358 & 1.3 \\
\hline 17 & 020801 & DeKalb & 4.30 & 0.000997 & 1.4 \\
\hline 18 & 960500 & Thomas & 18.58 & 0.00843 & 1.4 \\
\hline 19 & 021810 & DeKalb & 3.17 & 0.01047 & 1.4 \\
\hline 20 & 030303 & Columbia & 0.00 & 0.041 & 1.8 \\
\hline 21 & 030102 & Columbia & 0.00 & 0.114 & 1.8 \\
\hline 22 & 070104 & Henry & 0.00 & 0.493 & 1.8 \\
\hline 23 & 950100 & Taylor & 0.00 & 0.581 & 2.8 \\
\hline 24 & 960300 & Jeff Davis & 0.00 & 0.909 & 2.5 \\
\hline
\end{tabular}

${ }^{\mathrm{a}}$ Cluster numbers correspond to clusters in Figure 5.

${ }^{\mathrm{b}}$ Clusters are centered at the geographic centroid of the census tract listed

c The county in which the cluster's centroid falls is listed

${ }^{\mathrm{d}}$ Clusters with a 0 radius indicate a cluster localized to that specific census tract

${ }^{\mathrm{e}}$ Relative risk refers to the estimated risk within the cluster divided by the estimated risk outside the cluster

${ }^{\mathrm{f}}$ Note: Large relative risks should be interpreted with caution and may be artificially inflated due to small sample sizes

\section{DISCUSSION}

The purpose of this study was to examine melanoma incidence in GA over time and by race, SES, and gender. From 2000-2011, the burden of melanoma was larger among Whites than Blacks, which is consistent with previous studies (Shoo and Kashani-Sabet, 2009; Singh et al, 2011; American Cancer Society, 2013). Among darkerskinned persons, a high amount of melanin in the epidermis is protective against the development of ultraviolet (UV)induced melanoma (Shoo and Kashani-Sabet, 2009). Examination of incidence rates over time shows that melanoma diagnoses among Whites have been increasing in GA, reflective of trends in the US (Jemal et al, 2011). This increase has been ascribed to an increasing amount of natural (sun) and artificial (tanning beds) UV radiation (UVR) exposure and to increase in general cancer awareness and early detection, which may inflate incidence rates (Jemal et al, 2011).
Geographical disparities in GA were also observed among Whites and Blacks. Higher melanoma incidence rates for Whites were evident in the urban areas around the city of Atlanta and in the northeastern region of the state. Alternatively, high incidence rates for Blacks were seen in the more rural areas of GA in the northern and southern public health districts. Among patients in California and Massachusetts, individuals residing in urban areas had a greater number of incident melanoma cases compared to rural areas; however, there was no significant difference between incidences of early stage cancer by location (Blair et al, 2006; DeChello and Sheehan, 2006). Furthermore, studies in Austria and Sweden found higher melanoma incidence rates in urban districts relative to rural ones (DeChello and Sheehan, 2006; Haluza, et al, 2014). In contrast, in South Australia, patients living in rural areas had higher diagnoses of in situ melanomas but a lower proportion of invasive melanomas (Pérez-Gómez, 2008). Thus, most previous reports suggest that melanoma incidence rates are higher in urban areas, as was found for 
Whites in the current study. However, conflicting findings exist, and the limited evidence of the geographical distribution of melanoma incidence by racial subgroup underscores the relevance of the findings of the current study for Blacks.

Differences in melanoma incidence rates between rural and urban inhabitants have been attributed to a variety of factors. Screening is more readily available in urban areas compared to rural areas, leading to more diagnoses in those areas (DeChello and Sheehan, 2006). Further, differences in melanoma incidence rates may stem from behaviors of urban dwellers that encourage intermittent exposure to UVR, who may not protect themselves as well as rural inhabitants who receive relatively constant exposure (DeChello and Sheehan, 2006; Haluza, et al,, 2014; PérezGómez, 2008). According to the American Academy of Dermatology and Georgiacancerinfo.org websites, most of the free screening for skin cancer in GA is available in the urban areas around Atlanta, Savannah and Augusta. Additionally, the Environmental Protection Agency (EPA) has noted that GA receives high UV exposure relative to more northern states in the US, which can contribute to a high risk of developing melanoma (Wilkinson, Cameron, 2004). Thus, trends in GA are likely related to screening and to behavioral factors, which must be considered in developing public health interventions for at-risk populations. The present results suggest that improved access to screening in rural GA is needed, since the highest melanoma incidence rates for Blacks are found in those areas, and a large percentage of Blacks in GA are diagnosed at a late stage. Racially-sensitive screening and targeted educational programs in rural GA may improve the outcomes among the Black population.

In GA, individuals with high SES typically live in more urban areas (U.S. Census Bureau, 2010). For example, in Fulton County, which encompasses much of the city of Atlanta, the median household income is $\$ 56,313$, which is greater than the median household income for the state of GA, $\$ 47,469$. In this report, map comparisons of public health districts in GA are consistent with previous research findings that higher melanoma incidence rates are associated with high SES and with urbanicity for Whites and, to a lesser extent, for Blacks (Clarke et al, 2010; Risser and Miller, 2012; Hausauer et al, 2011). This relationship between high SES and melanoma diagnoses may be attributable to intermittent UV exposure during vacations and leisure time relative to those who receive more chronic exposure to UV, as was found through a study in Norway (Pollitt et al, 2012). The association of melanoma with UV radiation was also seen in California only among those living in the highest $40 \%$ of SES-ranked neighborhoods (Clarke et al, 2010). Furthermore, in Massachusetts, individuals with high SES were more likely to vacation in locations of increased sun exposure and to utilize tanning beds (DeChello and Sheehan, 2006). The complex interrelationships between urbanicity, and SES and subsequent impact on melanoma incidence and stage at diagnosis in GA warrants further attention.
The Getis-Ord Gi* Statistic hotspot analysis for melanoma incidence among individuals of all races indicated a statistically significant hotspot in the northeastern area of GA and two cold spots in the middle of the state. When this analysis was conducted for Whites only, a second hot spot was evident in the southwestern area, including Grady, Thomas, and Brooks Counties. There was also a cold spot of melanoma cases among Whites in the middle, southeastern region of GA. These hot and cold spots are consistent with the racial distribution seen among GA counties, where hot spots coincide with counties with high percentages of Whites, and cold spots have lower percentages of Whites. The analysis restricted to Whites shows a similar trend between high proportions of Whites and hot spots. These trends correspond with higher melanoma incidence rates among Whites compared to Blacks (Shoo and KashaniSabet, 2009; Singh et al, 2011; American Cancer Society, 2013). Due to the varied information provided for each, the Getis-Ord Gi* Statistic and SaTScan were utilized in this research to analyze clustering of melanoma incidence. The Getis-Ord method allows for visualization of hot spots and cold spots at the county level; the SaTScan analysis is more specific at the census-tract level. However, relative risks generated through SaTScan should be interpreted with caution because of small sample sizes within census tracts relative to county-level data. County-level data would have helped avoid sample size issues, but spatial patterns would have been less visible due to these larger sizes (Wagner et al, 2013).

The evident disparities in stage at diagnosis of melanoma between Whites and Blacks in GA present a public health challenge that requires attention. A primary difficulty in diagnosis among ethnic minorities lies in the atypical presentation of melanoma on sun-protected skin (i.e., acral, subungual and mucosal) with unknown etiology and no established lifestyle, occupational or environmental risk factors (Shoo and Kashani-Sabet, 2009). Since most melanoma cases in Blacks are detected at later stages, this population has poorer outcomes than Whites (Shoo, Kashani-Sabet, 2009). For patients with melanoma, latestage diagnosis (regional or distant) is associated with statistically significant lower 5-year survival rates; from $96 \%$ for localized melanoma to $61 \%$ for regional and $12 \%$ for distant disease (Gellar et al, 1996; Reyes-Ortiz, et al, 2005). Therefore, non-Whites are more likely to have lower melanoma-specific survival rates relative to Whites (Wu et al, 2011). Consistent with the findings of the present report, a recent survey in Florida identified clusters of late-stage melanoma diagnoses among neighborhoods with low SES and a high proportion of minority (Hispanic) residents $(\mathrm{Hu}$ et al, 2014). The present results demonstrate that screening programs for Whites may be most effective in Fulton, CobbDouglas and DeKalb public health districts; screening for Blacks should focus on Gainesville, Rome, and Valdosta public health districts.

As demonstrated by the present data, differences between melanoma incidence rates for White males and females vary depending on the geographic location. In general, countries with higher incidence rates, such as Australia and the US, have a greater proportion of male cases compared to 
countries with lower incidences, such as the United Kingdom, where there is a higher percentage of female cases (Giblin and Thomas, 2007; Garbe and Leiter, 2009). The disparities seen in melanoma incidences rates between White males and females in GA are similar to those found in the rest of the US (Jemal et al, 2011; Singh et al, 2011; Geller et al, 1996; DeChello and Sheehan, 2006; ReyesOrtiz, et al, 2005; Doben and MacGillivray, 2009; Linos et al, 2009; Nasseri, 2004). Although males had higher incidence rates, the geographical distribution was similar for both genders. Furthermore, for males and females, there were equal numbers of counties in which high incidence rates corresponded with high SES. There were insufficient data at the county level to compare Whites and Blacks by gender.

Some alternative patterns between melanoma and gender have been seen within the US. Among minority populations, females constitute a greater proportion of melanoma cases compared to Whites (Shoo and KashaniSabet, 2009; Wu et al, 2011). Additionally, in the US between 1999 and 2006, there were higher melanoma incidence rates among female adolescents and young adults aged 15 to 39 years compared to males of the same age (Weir et al, 2011). Overall, these differences between males and females could be attributed to genetics or behavioral patterns. There may be an association between female hormones and melanoma, especially when oral contraceptives or hormone-replacement therapy is involved (Weir et al, 2011). Further, young girls are more likely to participate in indoor and outdoor tanning behaviors that lead to high exposure to UV radiation (Weir et al, 2011). However, the higher incidence rates seen among males in GA could be attributed to occupational differences, with more outdoor jobs for males compared to females. Therefore, public health intervention programs in GA should be geared towards individuals who work outside to ensure that they protect themselves from UV radiation.

The strengths of this study include the comprehensiveness of the analysis among a variety of factors and the use of mapping tools for data visualization. The results reveal trends in melanoma incidence by race, gender, and SES. Illustrating these trends via GIS facilitated the interpretation of the geographic distribution of melanoma incidence in GA. In addition, use of pre-existing datasets to generate hypotheses on overall trends identifies populations at risk for melanoma. Finally, this is the first descriptive epidemiologic study of melanoma in GA that identifies areas that need to be targeted by public health interventions. Limitations of this study involve the low numbers of melanoma cases among Blacks at the county level and the challenges of determining SES. Since melanoma diagnoses are rare among Blacks, disparities by race were analyzed at the public health district level rather than the county level, which would have provided a finer level of geographic specificity. However, patterns detected at the public health district level may be practically applicable because public health interventions would likely be coordinated at the district level, since local health districts engage more with the community. Further, SES is a complicated construct, involving a variety of factors other than income, which need to be considered in future studies. The previously published studies utilized a variety of factors to determine SES, including percentage of high school graduates, median household income, employment types, and marital status (Aase and Bentham, 2010; Aarts et al, 2010; Van der Aa et al, 2011; Clegg et al, 2009; Pollitt et al, 2012; Mandalà et al, 2011; Youl et al, 2011; Zell et al, 2008; Geller et al, 1996). Comprehensive indices, such as the social vulnerability index, may provide additional insight into these patterns and future studies should strive to evaluate melanoma in relation to these or similar metrics (Cutter et al, 2003). Additionally, as a descriptive, ecological study, conclusions about causality between melanoma incidence and SES or urbanicity cannot be inferred, and findings should be interpreted with caution. Future studies should examine individual-level data to evaluate the relationship between melanoma incidence and risk factors, while controlling for confounding variables. Findings from the current study inform these studies, and suggest that they should be conducted in urban areas around Atlanta among Whites and in the more rural northern and southern public health districts among Blacks.

The differences in geographic distribution of high melanoma incidence rates among Blacks and Whites have interesting public health implications for prevention strategies. For example, the present results suggest that interventions geared towards Blacks should be located in more rural areas in GA; those for Whites should be more concentrated in urban areas. Further studies will examine reasons for these geographical disparities, such as the accessibility of screening in urban versus rural areas and the impact it may have on treatment. Identification of specific populations in GA at higher risk for melanoma will also help target prevention and education efforts. Currently, the Georgia Cancer Coalition publishes general demographic and geographic information on all cancers and the Georgia Comprehensive Cancer Registry collects information on cancer cases, but there is no statewide or district-wide public health prevention effort specifically targeted towards melanoma.

\section{IMPLICATIONS FOR PUBLIC HEALTH}

In GA, Whites have a higher burden of melanoma relative to Blacks; however, Blacks are more often diagnosed at later stages, and those with melanoma are more likely to have higher mortality rates. Furthermore, among Whites, males have higher incidence rates relative to females. These higher rates are associated with high SES as well as geographic locations: northern and urban regions for Whites and rural regions for Blacks. Ultimately, based on these trends, public health interventions should focus on Whites in and around Atlanta and Blacks in northern and southern rural areas, especially those with high SES.

\section{Acknowledgement}

The authors would like to thank the GCCR for their assistance and guidance with cancer data collection and analysis. 


\section{References}

Aarts MJ, van der Aa MA, Coebergh JWW, Louwman WJ. Reduction of socioeconomic inequality in cancer incidence in the South of the Netherlands during 1996-2008. Eur J Cancer. 2010; 46(14): 2633-46.

Aase A, Bentham G. Gender, geography and socio-economic status in the diffusion of malignant melanoma risk. Soc Sci Med. 1996; 42(12): 1621-37.

American Cancer Society. Cancer Facts \& Figures 2013.

Blair SL, Sadler GR, Bristol R, Summers C, Tahir Z, Saltzstein SL. Early cancer detection among rural and urban Californians. BMC Public Health. 2006; 6:194.

Boscoe F, Johnson C, Sherman R, Stinchcomb D, Lin G, Henry K. The Relationship Between Area Poverty Rate and Site-Specific Cancer Incidence in the United States. Cancer. 2014; 2191-8.

Clarke CA, Moy LM, Swetter SM, Zadnick J, Cockburn MG. Interaction of area-level socioeconomic status and ultraviolet radiation on melanoma occurrence in California. Cancer Epidemiol Biomarkers Prev. 2010; 19(11): 2727-33.

Clegg LX, Reichman ME, Miller BA, et al. Impact of socioeconomic status on cancer incidence and stage at diagnosis: selected findings from the surveillance, epidemiology, and end results: National Longitudinal Mortality Study. Cancer Causes Control. 2009; 20(4): 417-35.

Cutter SL, Boruff BJ, Shirley WL. Social vulnerability to environmental hazards. Social Science Quarterly. 2003; 84(2): 242-61.

DeChello LM, Sheehan TJ. The geographic distribution of melanoma incidence in Massachusetts, adjusted for covariates. Int Journal Health Geogr. 2006; 6:194.

Doben AR, MacGillivray DC. Current concepts in cutaneous melanoma: Malignant melanoma. Surg Clin N Am. 2009; 89: 713-25.

Environmental Protection Agency (EPA). Monthly average UV Index. http://www.epa.gov/sunwise/uvimonth.html

Environmental Systems Research Institute (ESRI), (2014). ArcGIS Desktop Help 10.1.

Garbe C, Leiter U. Melanoma epidemiology and trends. Clin Dermatol. 2009; 27: 3-9.

Geller AC, Miller DR, Lew RA, et al. Cutaneous melanoma mortality among the socioeconomically disadvantaged in Massachusetts. Am J Public Health. 1996; 86(4): 538-43.

Giblin AV, Thomas JM. Incidence, mortality and survival in cutaneous melanoma. J Plast, Reconstr Aesthet Surg. 2007; 60: 32-40.

Haluza D, Simic S, Moshammer H. Temporal and spatial melanoma trends in Austria: An ecologic study. Int J Environ Res Public Health. 2014; 11: 734-48.

Harvey V, Patel H, Sandhu S, Wallington S, Hinds G. Social determinants of racial and ethnic disparities in cutaneous melanoma outcomes. Cancer Control. 2014; 21 (4): 343-9.

Hausauer AK, Swetter SM, Cockburn MG, Clarke CA. Increases in melanoma among adolescent girls and young women in California: Trends by socioeconomic status and UV radiation exposure. Arch Dermatol. 2011; 147(7): 783-9.

$\mathrm{Hu}$ S, Sherman R, Arheart K, Kirsner R. Predictors of neighborhood risk for late-stage melanoma: Addressing disparities through spatial analysis and area-based measures. $J$ Invest Dermatol. 2014; 134: 937-45.

Jemal A, Saraiya M, Patel P, et al. Recent trends in cutaneous melanoma incidence and death rates in the United States, 19922006. J Am Acad Dermatol. 2011; 65(5): S17.e1- S17.e11.

Kulldorff M. SaTScan User Guide for version 9.4. http://www.satscan.org/. February, 2015.
Linos E, Swetter SM, Cockburn MG, et al. Increasing burden of melanoma in the United States. J Invest Dermatol. 2009; 129(7): 1666-74.

Mandalà M, Imberti GL, Piazzalunga D, et al. Association of socioeconomic status with breslow thickness and disease-free and overall survival in stage I-II primary cutaneous melanoma. Mayo Clin Proc. 2011; 86(2): 113-9.

Nasseri K. Secular trends in cancer mortality, California 19701998. Cancer Detection and Prevention. 2004; 28: 143-54.

Office of Air and Radiation. Georgia melanoma facts: May 2010. www.cdc.gov/cancer/skin.

Pérez-Gómez B, Aragonés N, Gustavsson P, Lope V, LópezAbente G, Pollán M. Socio-economic class, rurality and risk of cutaneous melanoma by site and gender in Sweden. BMC Public Health. 2008; 8:33.

Pollitt RA, Swetter SM, Johnson TM, et al. Examining the pathways linking lower socioeconomic status and advanced melanoma. Cancer. 2012; 118(16): 4004-13.

Reyes-Ortiz CA, Goodwin JS, Freeman JL. The effect of socioeconomic factors on incidence, stage at diagnosis and survival of cutaneous melanoma. Med Sci Monit. 2005; 11(5): RA163-72.

Risser DR, Miller EA. Cancer in relation to socioeconomic status: Stage at diagnosis in Texas, 2004-2008. South Med J. 2012; 105(10): 508-12.

Shoo BA, Kashani-Sabet M. Melanoma arising in African-, Asian-, Latino and Native-American populations. Semin Cutan Med Surg. 2009; 28(2): 96-102.

Singh SD, Ajani UA, Johnson CJ, et al. Association of cutaneous melanoma incidence with area-based socioeconomic indicators United States, 2004-2006. J Am Acad Dermatol. 2011; 65 (5 Suppl 1): S58-68.

U.S. Census Bureau; Census 2010; Quick Facts; Using American FactFinder http://factfinder2.census.gov.

Van der Aa MA, de Vries E, Hoekstra HJ, Coebergh JWW, Siesling S. Sociodemographic factors and incidence of melanoma in the Netherlands, 1994-2005. Eur J Cancer. 2011; 47(7): 1056-60.

Wagner SE, Bauer SE, Bayakly AR, Vena JE. Prostate cancer incidence and tumor severity in Georgia: Descriptive epidemiology, racial disparity, and geographic trends. Cancer Causes Control. 2013; 24(1): 153-66.

Weir HK, Marrett LD, Cokkinides V, et al. Melanoma in adolescents and young adults (ages 15-39 years): United States, 1999-2006. J Am Acad Dermatol. 2011; 65 (5 Suppl 1): S38-49.

Wilkinson D, Cameron K. Cancer and cancer risk in south Australia: What evidence for rural-urban health differential? Aust J Rural Health. 2004; 12: 61-6.

Wu X-C, Eide MJ, King J, et al. Racial and ethnic variations in incidence and survival of cutaneous melanoma in the United States, 1999-2006. J Am Acad Dermatol. 2011; 65(5): S26.e1S26.e13.

Youl PH, Baade PD, Parekh S, et al. Association between melanoma thickness, clinical skin examination and socioeconomic status: Results of a large population-based study. Int J Cancer. 2011; 128(9): 2158-65.

Young JL Jr, Roffers SD, Ries LAG, Fritz AG, Hurlbut AA (eds). SEER summary staging manual - 2000: Codes and coding instructions, National Cancer Institute, NIH Pub. No. 01-4969, Bethesda, MD, 2001.

Zell JA, Cinar P, Mobasher M, et al. Survival for patients with invasive cutaneous melanoma among ethnic groups: The effects of socioeconomic status and treatment. J Clin Oncol. 2008; 26(1): 66-75. 